\title{
Hacia la Construcción del Observatorio por la Equidad Territorial, de Género y Etaria en Xalapa, Veracruz, la Mirada Infantil ${ }^{1}$
}

\author{
L.Mendoza² - K. E. Carrillo ${ }^{3}$
}

\begin{abstract}
RESUMEN
Presentamos el avance de una investigación orientada a la creación del Observatorio para la Equidad Territorial, de Género y Etaria en Xalapa, Veracruz. Este trabajo forma parte de una red de investigaciones y proyectos desarrollados en el taller del último año de la carrera de arquitectura, con el propósito de visibilizar las desigualdades existentes entre las diferentes zonas de la ciudad y entre los grupos sociales y etarios para quienes la ciudad no es accesible de manera equitativa.

Uno de esos grupos sociales en quienes no se piensa al planear y construir la ciudad, lo conforman los niños y las niñas. Es por ello que en esta ocasión nos centramos en investigar, en diversos planteles educativos de Xalapa, la percepción acerca de los barrios y escuelas que habitan, la interrelación con los compañeros y con otros grupos sociales, y la idea de ciudad que ellos imaginan.
\end{abstract}

\begin{abstract}
We present the preliminary study of a research aimed to set up an Observatory on Territorial, Gender, and Age Equity in the city of Xalapa, Veracruz. This project belongs to a research and projects network developed around the senior year of the architecture career; this observatory will highlight the existing inequalities between the different areas of the city as well as those existing between different social or even age groups, for whom the city is not equally accessible.

One of these groups, which planners and city builders don't think of, consists of boys and girls. That is why the research presented here focuses on the perception that they have about the neighborhoods and schools they inhabit, the interrelation with other children, with other social groups, and the idea of the city they imagine.
\end{abstract}

Palabras Clave: infancia, hacer ciudad, equidad

Keywords: childhood, city making, equity

\footnotetext{
1 Para mayor detalle del proceso, materiales y resultados, ver Mendoza y Carrillo, (2017). Identidades Infantiles, Percepción del Barrio y la Ciudad en Niños de Diferentes Contextos. Congreso Internacional Arquitectonics: Mente, Territorio y Sociedad. https://pa.upc.edu/ca/Varis/altres/arqs/congresos/international-conference-arquitectonics-mind-land-society/final-papers/laura-mendoza-kaplan-karla-elisa-carrillo-rodriguez.pdf/view

2 Docente y Miembro del SIN 1. Facultad de Arquitectura de la Universidad Veracruzana. lamendoza@uv.mx, lameka57@yahoo.com.mx http://colectivobcc.wix.com/colectivobcc

3 Becaria del Sistema Nacional de Investigadores SNI, Facultad de Arquitectura de la Universidad Veracruzana. elisacarrillo93@gmail.com http://colectivobcc.wix.com/colectivobcc
} 


\section{Introducción}

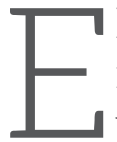
1 Observatorio por la Equidad Territorial, de Género y Etaria en Xalapa fue pensado desde hace más de una década en el taller de proyectos D-208 de la Facultad de Arquitectura de la Universidad Veracruzana (FAUV-Xalapa) y desde entonces, dentro de sus planes de clase, se ha ido construyendo un proceso de enseñanza de la arquitectura, en torno a la participación comunitaria dentro y fuera del aula, vinculando los proyectos de los estudiantes, en mayor o menor medida, con los habitantes de los diferentes barrios y comunidades.

La motivación principal ha sido evidenciar que la ciudad no es única ni homogénea y que si bien esto debe ser reconocido como una cualidad y a la vez como un potencial para desarrollar proyectos con identidad, también es cierto que encubre una terrible desigualdad de oportunidades de disfrute de la vida cotidiana, dependiendo de la edad, el género, la zona de la ciudad en que se viva y muchos otros factores físicos, sociales y económicos, que pensamos, no deberían ser limitantes.

Con el observatorio pretendemos evidenciar a los ojos de los estudiantes, investigadores, autoridades, y de los habitantes mismos, estas desigualdades, para estar en posibilidad de reinventar la ciudad de manera más equitativa en cuanto a vivienda, y equipamiento para la educación, la salud, el comercio, el deporte, el arte, etc., a través de revaluar las diferentes miradas de cada uno de los grupos sociales y etarios, de manera que todas sean tomadas en cuenta para la mejora continua de las ciudades.

La estrategia para contactar y reconocer a los diferentes habitantes del barrio ha sido la realización de talleres comunitarios; entre el 2001 y el 2015 se desarrollaron talleres comunitarios en diferentes sitios de la ciudad de Xalapa, en otros asentamientos en el estado y eventualmente en otros estados y países. En todos ellos se puso de manifiesto la importancia de la mirada desde la infancia acerca de la idea de ciudad: recordada por adultos y adultos mayores, vivida por niños, o imaginada y deseada para sus futuros hijos por los adultos jóvenes, siempre como motor para dar forma a nuevas maneras de vivir y convivir en las ciudades.

Los hallazgos así obtenidos nos llevan a investigar sobre las percepciones de niños y niñas, este grupo invisible hasta hace muy poco en la toma de decisiones de la ciudad. Francesco Tonucci (2016) plantea que "si una ciudad es buena para los niños, lo es para todos", lo cual se constituye en una guía sobre la cual caminar.

Paralelamente al taller de proyectos, las profesoras, algunos de los estudiantes y ex alumnos del taller nos hemos constituido como Colectivo Barrio, Ciudad y Convivencia ${ }^{4}$, con la intención de desarrollar investigación extracurricular, como los talleres "Identidades Infantiles" 5 que aquí se describen.

\section{Determinación de los sitios de estudio, el con- texto local}

El modelo de ciudad mexicana es el de una ciudad centralizada, con un área relativamente pequeña donde se concentran todos los servicios. En este modelo de ciudad, los servicios se difuminan en la medida en que se alejan del centro, hasta llegar, por un lado, a una periferia de más o menos pobreza, casi carente de los mismos, y por otro, a una periferia de riqueza auto-segregada y aislada, a la que se le ha dado el nombre de "suburbios" o "fraccionamientos cerrados", que los tiene a su alcance mediante el auto particular.

Con base en esta división, se definieron en Xalapa las 4 áreas donde desarrollaríamos los nuevos talleres, de manera que nos pudieran dar resultados para su comparación y análisis. Hasta ahora se había trabajado preferentemente en el espacio público abierto, pero considerando la situación de inseguridad que impera,

4 http://colectivobcc.wix.com/colectivobcc

5 Con la participación de Karla Elisa Carrillo Rodríguez, Andrés David López Gómez, Julián Emanuelle Maldonado Vázquez, Laura Mendoza Kaplan, Jorge Francisco Morales Guerra y Paola Kristell Ramírez Prieto. Colectivo Barrio, Ciudad y Convivencia, 2017. 
se optó por realizarlos en escuelas primarias, tanto privadas como públicas, para analizar en ellas, la percepción que tienen los niños, de su contexto inmediato. Ver figura 1.

\section{Proceso de investigación}

El proceso fue definido con base en las estancias académicas ${ }^{6}$ con Josep Muntañola7, quien desarrolla desde los años 70 un material con bloques de madera que permite sistematizar las respuestas de los niños; así mismo, en el Taller Con y Para los Niños, realizado en el seno del Congreso Arquitectonics ${ }^{8} 2016$, en el curso-taller de la Red de Ciudades Amigas de la Infancia (Red CAI), y a partir de la colaboración con Sergi Méndez?

En nuestro caso, la investigación ligada al observatorio de equidad está encaminada a mapear la percepción que tienen los niños de su barrio: si lo ven igual los niños que las niñas y si perciben igual su barrio y su ciudad un grupo de niños en el centro de la ciudad, en la ciudad consolidada o en las periferias; si se trata de las periferias auto-generadas, de grupos sociales con escaso poder adquisitivo, en la periferia "institucional" (INFONAVIT, FOVISSSTE, etc.) o en los fraccionamientos cerrados de las periferias, con personas de alto poder adquisitivo.

Dinámica del taller

Se arma el equipo de 5 integrantes: moderador, 2 fotógrafos, un "reportero" y un dibujante.

Se solicita un grupo de 6 niños, preferentemente 3 niñas y 3 niños.

Se delimita un área lisa de 2 por 2 metros en el patio, para que trabajen dentro de ella.

1. El moderador les pide que entre todos construyan el barrio donde está su escuela.

Mientras ellos construyen, el fotógrafo 1 toma detalles de lo que van construyendo; el fotógrafo 2 toma, desde un mismo ángulo, fotos cada 30 segundos, para evidenciar el trabajo colaborativo, o ausencia de él; y el reportero anota todo lo que digan los niños y niñas.

2. Al término del tiempo establecido, se les

Figura 1. Plano de la ciudad de Xalapa, delimitando las 4 áreas de estudio. Elaboración: Colectivo Barrio Ciudad y Convivencia, 2016.

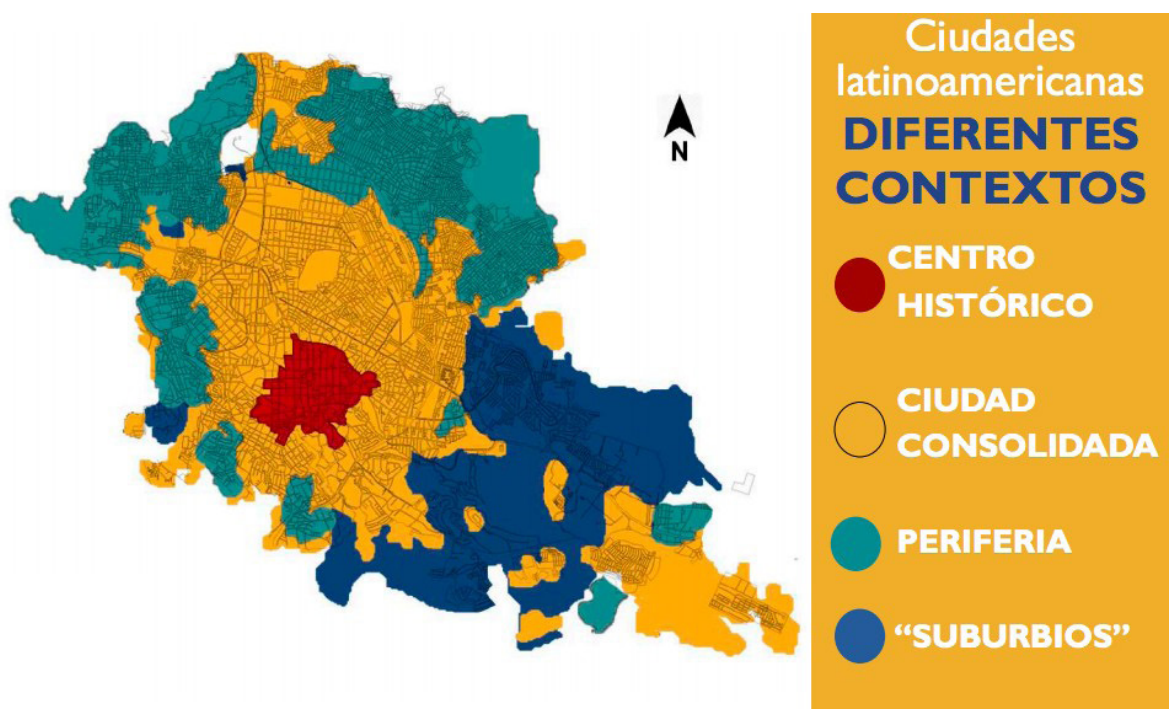

6 Septiembre 2015-junio de 2016, y mayo-junio de 2017.

7 Catedrático emérito de la UPC, autor de La Arquitectura Como Lugar (1974), donde describe este material.

8 Co diseñado por Josep Muntanyola y Laura Mendoza Kaplan, y coordinado por esta última.

9 Mendez, Sergi (2018). La diagnosi arquitectònica de l'espai públic a través de la percepció dels infants (La diagnosis arquitectónica del espacio público a través de la percepción de los niños. Tesis doctoral UPC. 
pide que expliquen lo que hicieron.

El fotógrafo 1 toma una foto instantánea para que el dibujante marque qué es cada espacio. El reportero anota los comentarios.

El moderador hace preguntas que nos ayuden a comprender aspectos que no queden claros en su exposición. Por ejemplo, por qué pusieron un auto descompuesto en la calle y cómo se sienten al pasar por donde está.

3. Se les pide ahora, que construyan cómo les gustaría que fuera su barrio en unos años, cómo lo mejorarían.

4. Al finalizar el tiempo, exponen, se hacen preguntas y se da por terminada la actividad.

Figura 2. Talleres comunitarios utilizando bloques de madera.

Niños de la primaria Pedro de Gante y Colectivo Barrio Ciudad y Convivencia, 2017

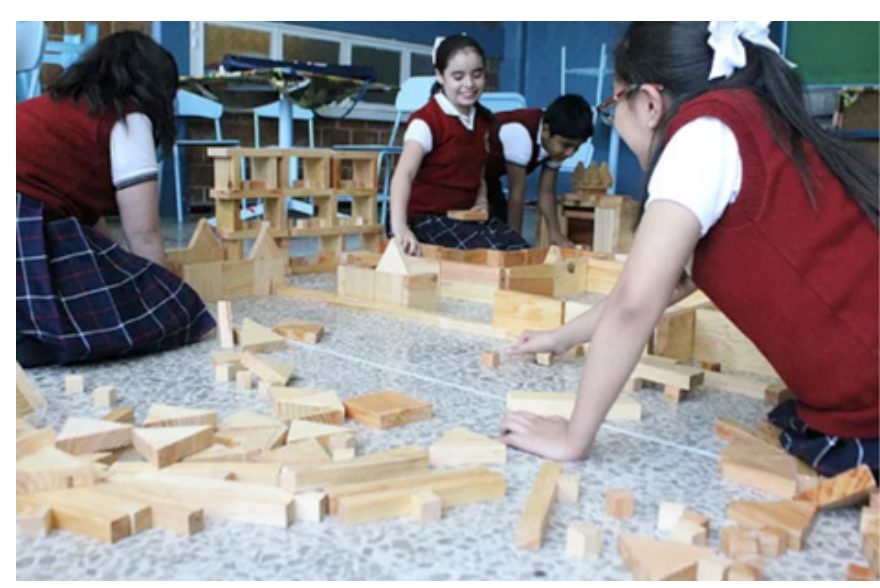

\section{Avances}

En este primer muestreo piloto se tuvo acceso a 4 escuelas públicas y 6 del sector privado, que trabajan según diferentes tipos de enseñanza -oficial o alternativo-, y que se ubican en 3 de los distintos sectores de la ciudad de Xalapa, Veracruz; no tuvimos acceso a los fraccionamientos cerrados o suburbios.

A continuación, mostramos diferentes hallazgos, que nos ayudarán a esclarecer las estrategias definitivas para el mapeo en toda la ciudad, así como a la conformación de los indicadores que den base al Observatorio propuesto, y que sirva de base para la elaboración de proyectos de intervención, de diversas índoles, no exclusivamente arquitectónicas.

\section{a) Construcción del barrio}

En escuelas privadas del Centro Histórico destaca que los niños realmente reconocen los espacios que rodean su escuela, pese a que un 80\% de ellos no viven en las calles o colonias cercanas a la institución.

En la figura 3 puede observarse como, aunque con algunas variaciones en distancias y proporciones, los edificios-hito de la maqueta coinciden con lo que existe y su ubicación en el contexto físico real. Así mismo es de notar que perciben la zona como segura y alegre.

En las escuelas que se ubican en la ciudad

Figura 3. Reconstrucción digital de la maqueta, realizada por los niños de un colegio privado, ubicado en el Centro de la Ciudad de Xalapa, Ver. y de la realidad, con base en un plano de google maps. Mendoza y Carrillo 2017.

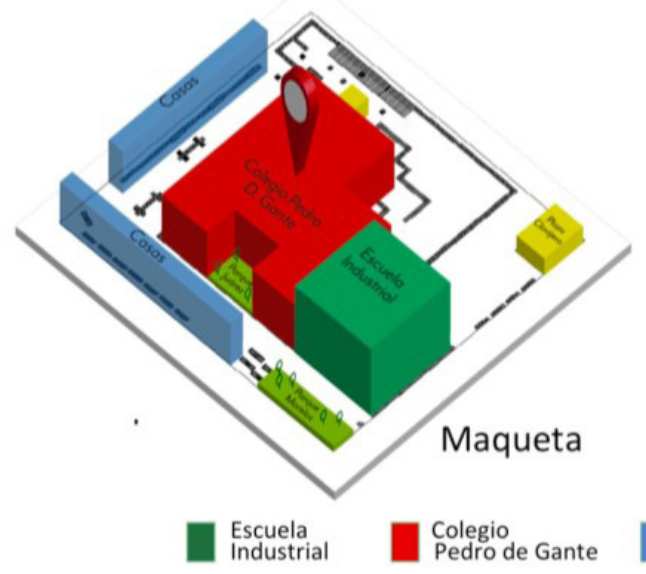

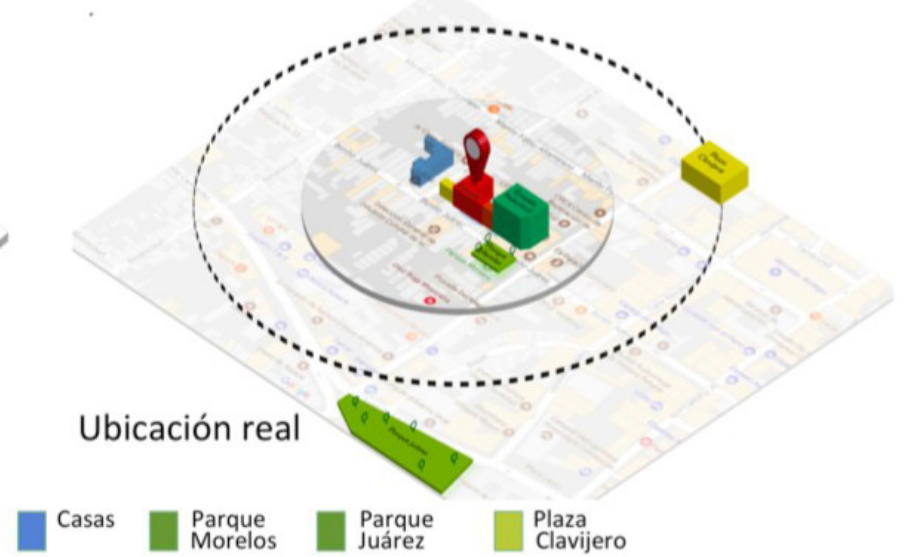


consolidada y en la periferia, existe el caso contrario: la mayoría de los niños vive cerca de la escuela por lo que su relación con el contexto es mayor, sin embargo, no lo representa con certeza; le falta representar algún equipamiento, o lo ubica en diferente sector de su barrio.

Lo que sí queda muy claro, es que perciben inseguridad en el medio en el que viven; esta situación es algo que preocupa a los niños y niñas, y claramente la quieren erradicar, de una forma u otra, en su "barrio ideal".

\section{b) Construcción del barrio ideal}

En el barrio ideal destacaron dos tipos de ciudad:

En las escuelas de la periferia, el barrio ideal fue representado por una casa grande, creando "vecindarios" entre los niños del salón o resguardándose de los "malos" con una ciudad amurallada. Los sitios que desean incorporar son lugares de culto (iglesias) y refugios para los perros callejeros. En el centro histórico y la ciudad consolidada, el ideal de ciudad no solo se basa en tener una casa para vivir y resguardarse, sino en tener equipamiento urbano para la vida cotidiana, y establecimientos para la compra y venta de productos.

En la figura 5 se representan los comentarios vertidos en los diferentes talleres; el diámetro del círculo refleja la frecuencia con que se mencionó cada tipo de equipamiento. El 100\% de los equipos mencionan áreas para jugar. El 87\% construye viviendas, las suyas u otras "más grandes" o "más bonitas". El 75\% incluye en su ciudad ideal, a su propia escuela y algún tipo de hospital. El 62\% nombra restaurantes, especialmente pizzerías. El 50\% menciona algún tipo de comercio: centros comerciales, tienditas de la esquina, etc., y considera refugios para animales, especialmente perros. En materia de transporte, el 25\% se preocupó por hacer accesible el transporte para todos y $37.5 \%$ hizo mucho énfasis en no querer autos por no ser "saludables" o por contaminar la ciudad.

Figura 4. Tabla comparativa de espacios representados en la maqueta ideal.

\begin{tabular}{|c|c|c|}
\hline $\begin{array}{c}\text { El Barrio en el que me gustaría } \\
\text { vivir }\end{array}$ & $\begin{array}{c}\text { En el Centro Histórico y Ciudad } \\
\text { Consolidada }\end{array}$ & En la Periferia \\
\hline Espacios educativos & Sí (primaria, kínder, secundaria). & Sí (primaria). \\
\hline Áreas para recreación & $\begin{array}{c}\text { Parque, zoológico, museo, gale- } \\
\text { ría, plaza comercial, alberca y } \\
\text { área para acampar. }\end{array}$ & Parque, playa, volcán. \\
\hline Áreas para comer & Pizzería, sushi, restaurant. & No \\
\hline Áreas de salud & Hospital, clínica de salud. & No \\
\hline Transporte Público & $\begin{array}{c}\text { Ruta de tren, bicicletas, cami- } \\
\text { nar. }\end{array}$ & No \\
\hline Área religiosa & Iglesia & Una barda. \\
\hline Barrera (delimitación) & No & No \\
\hline Abasto & Un supermercado, plaza co- \\
\hline mercial, farmacias. & Sí & Sí \\
\hline
\end{tabular}

10 Mendoza y Carrillo, (2017). Op.cit. 
Figura 5. Análisis cualitativo de los espacios. Resumen de comentarios mientras construían sus maquetas. Mendoza y Carrillo, 2017.

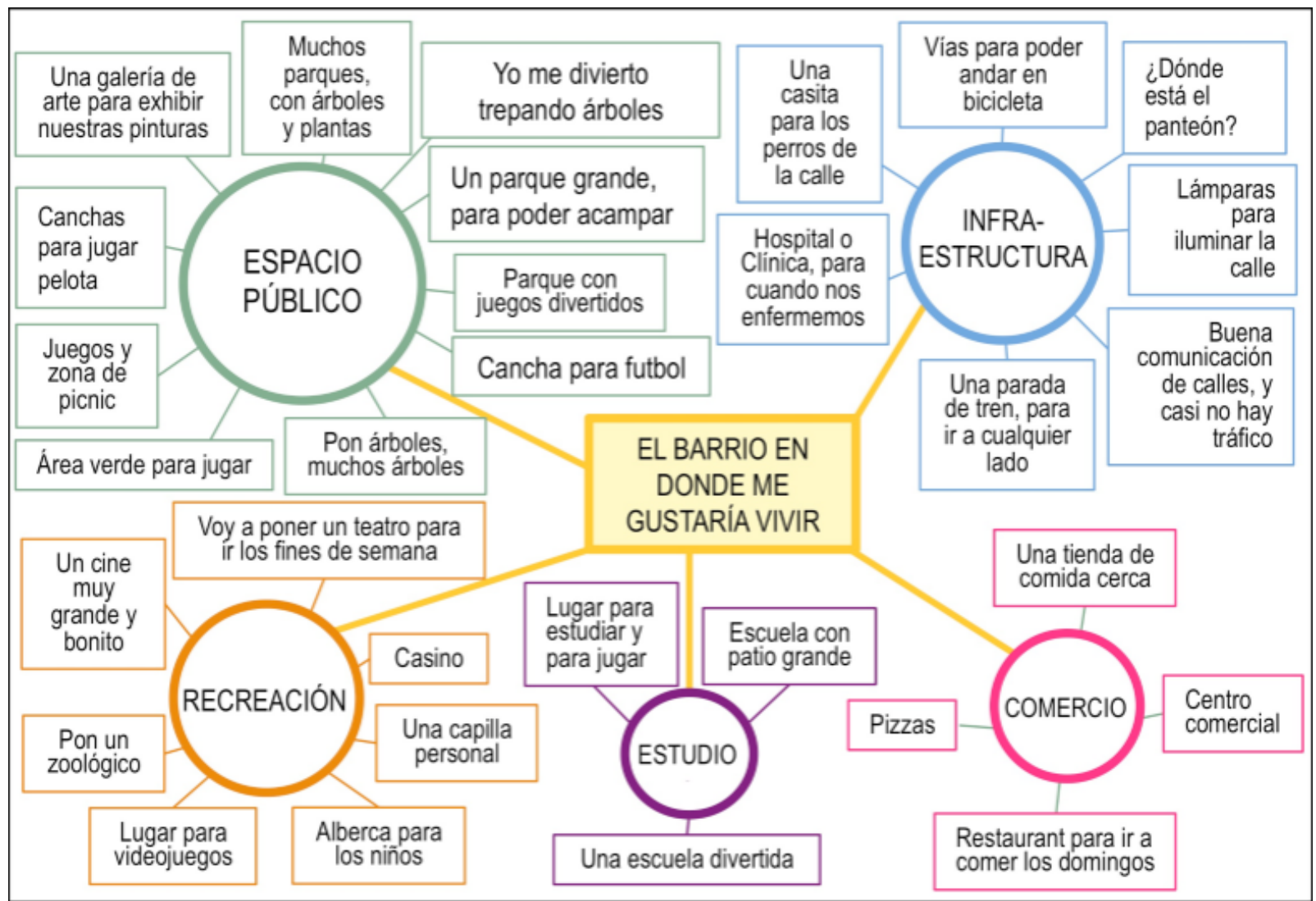

Otros aspectos analizados ${ }^{10}$ fueron: Interacción

A través de la secuencia de fotos, registramos el tiempo en el que los niños trabajan de manera integrada, y aquellos que trabajan de manera aislada. En la zona central, el 90\% del tiempo realizaban la maqueta entre todos; en la zona consolidada de la ciudad, el 68\% del tiempo trabajaban de forma colaborativa; y en la periferia de la ciudad solo el 55\% del tiempo colaboraban.

\section{Género}

Relacionando la interacción entre niños y niñas, pudimos observar que en la zona central, en los 3 talleres trabajaron integrados por género, en la zona consolidada, en 2 de 3 talleres, y en la periferia, en una escuela trabajaron integrados y en la otra separados por género. Liderazgo

En la zona central en 2 talleres había 2 líderes, un niño y una niña, y en el otro, solo una niña. En la zona consolidada había una líder niña; en la periferia, en un caso fue un niño el líder, y en el otro no había líder, trabajaron de manera independiente.

\section{Calidad de los espacios urbanos}

Luego de digitalizar las maquetas en planta, se realizó un análisis espacial mediante el programa Sintaxis Espacial ${ }^{11}$, el cual arroja información sobre las áreas donde existe mayor grado de convivencia.

En este análisis, los colores de la izquierda, corresponden a las zonas de la ciudad, mostradas en la figura 1. Las manchas negras son las construcciones de los niños; la graduación

11 Los modelos de sintaxis espacial se iniciaron en 1984 con el trabajo clásico de Bill Hillier y Julienne Hanson The social logic of space. Ver más en https://sintaxisespacial.com/2014/12/21/space-syntax-bill-hillier-et-al/ 
Figura 6. Análisis mediante Sintaxis espacial. Jorge Morales, Colectivo BCC, 2017.

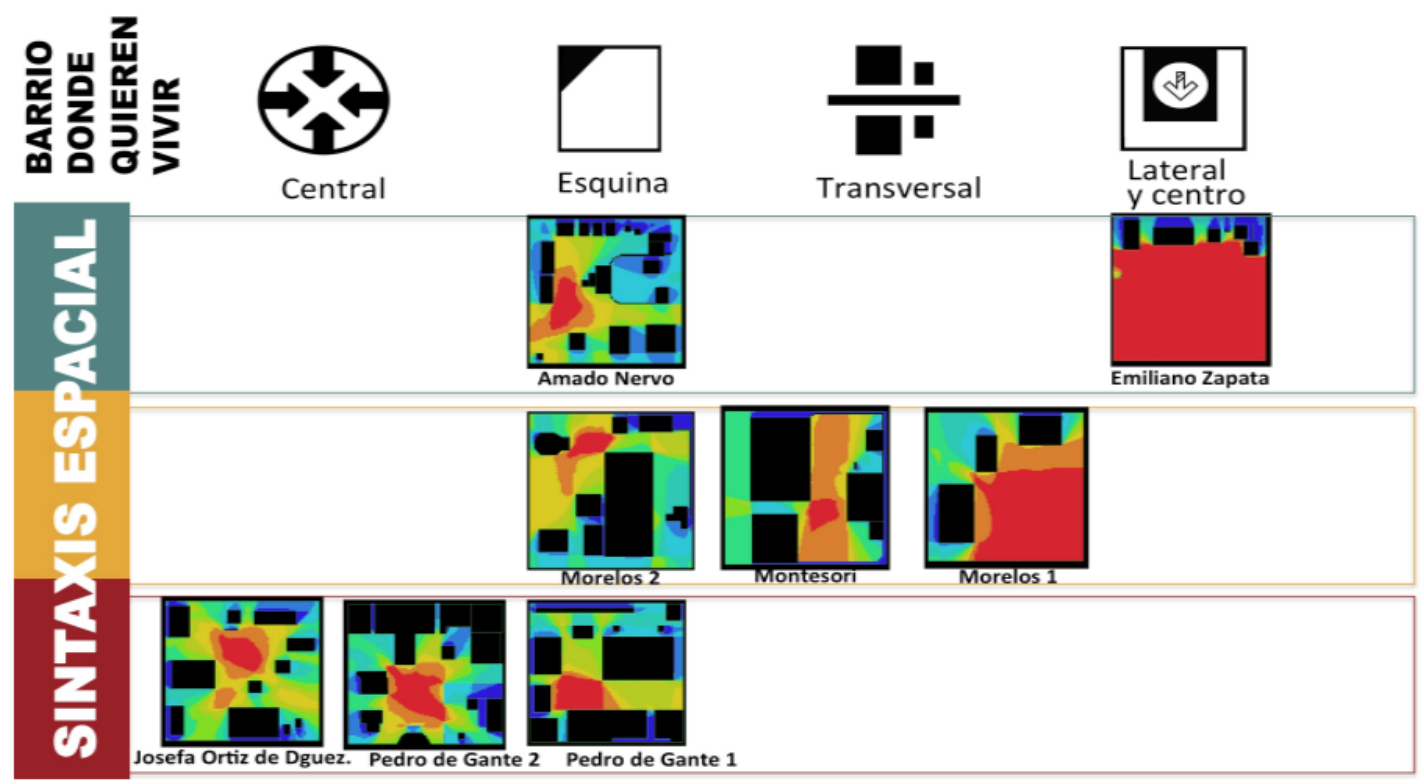

de color que arroja el análisis va del azul, que representa zonas que inhiben la interacción humana, y las zonas rojas son las áreas más propicias para la convivencia; entre más centralizada esté el área de reunión, se supondría el barrio más equitativo y por tanto, de mayor calidad espacial. Relacionando el trabajo colaborativo entre los niños, y la calidad espacial del barrio que diseñan, la tabla es evidente: donde hay colaboración el 100\% del tiempo (centro), generaron los mejores barrios.

\section{Reflexiones}

Esta metodología nos permite averiguar de manera amable y segura, desde un espacio de confianza -la escuela-, la mirada de los niños y niñas, propiciando la convivencia y la interacción entre ellos, de manera que se constituye, en sí, una forma de darse cuenta y mejorar por ellos mismos las situaciones que les afectan.

Para los estudiantes de arquitectura, estos talleres han dado pie a proyectos de fin de carrera y han servido de fundamento para incluir la mirada de género y de equidad territo- rial y etaria en sus proyectos. Particularmente, el taller que aquí se describe ha sido un primer contacto con el barrio centrado en la mirada infantil, que les permite un conocimiento básico en cuanto a percepción, necesidades y aspiraciones de este grupo social; les habla también de diferencias territoriales muy marcadas en cuanto a desigualdades de género e integración entre niños y niñas en sus procesos educativos, y pone en claro necesidades barriales de infraestructura y equipamiento cuya satisfacción pueda contribuir a la construcción de un barrio seguro y amable con los niños y con todos los habitantes.

Para una siguiente etapa se tiene pensado realizar talleres de co-diseño para la escuela y el barrio, donde planeamos incluir el trabajo integrador, no sólo entre los niños y niñas, sino entre ellos y sus maestros y familias.

Finalmente, un dato al margen de esta investigación fue que, pese a que existe la legislación que restringe la inscripción a escuelas primarias a aquellas próximas al lugar de residencia $^{12}$, a las escuelas del centro no asisten niños que necesariamente viven en el mismo. Si bien creemos que esto contribuye al problema de

12 Según la Secretaría de Educación Pública en México, los niños deben inscribirse en una escuela próxima a su residencia. (http://calendariosep.mx/preinscripciones-sep-2016-a-primaria-requisitos/). 
la movilidad vehicular de la ciudad, no parece impactar desfavorablemente en la percepción de la ciudad, en la interrelación entre los compañeros de clase, ni en la calidad de espacios que los niños y las niñas proponen. Habrá que investigar, mediante otro tipo de taller, lo que sucede en su lugar de residencia.

Hacia la Construcción del Observatorio por la Equidad de Género, Territorial y Etaria en Xalapa, Veracruz, la Mirada Infantil.

Este mapeo piloto ha permitido sentar una primera base para conformar los indicadores que fundamenten la creación del observatorio propuesto, con vías a que sirva a su vez, tanto a proyectos universitarios de mejoramiento barrial como a la inclusión de la visión infantil, y que las medidas que de ella emerjan puedan incluirse en políticas públicas, a fin de llevarlas a la totalidad del territorio. Para esto es necesario definir indicadores cualitativos relacionados con la calidad de vida que permitan avanzar tanto en la comprensión como en la medición de la percepción y de las aspiraciones particulares de los infantes; que incluyan no sólo la satisfacción de necesidades físicas, sino también elevar los niveles de "bienestar" o del "buen vivir". En este contexto, la investigación aporta a la comprensión de los elementos que conforman el bienestar físico, social y psicológico que perciben y al que aspiran los niños y las niñas de los diferentes barrios de la ciudad analizada.

El conocimiento de su barrio permite ver qué tanto interactúan en él y cómo se sienten con respecto a ese hecho; qué zonas consideran peligrosas o seguras y por qué; y cómo perciben los problemas ambientales y cómo los afectan. La construcción de su barrio ideal, paradójicamente, pone en evidencia las carencias físicas y los problemas que les ocasionan.

Al mismo tiempo, el taller investiga aspectos que permiten comprender el nivel de bienes- tar social, al visibilizar el grado de interacción entre niños y niñas, lo que puede ser reflejo de relaciones desiguales entre géneros, en casa y en la escuela, y que podría estar vinculado a la violencia de género que aqueja a nuestra ciudad y en especial a algunas de sus zonas. La interacción relacionada con el liderazgo pretende explorar el tipo de barrio que se genera en un equipo como suma de individualidades ajenas entre sí, de individualidades en colaboración, o dirigido por uno o dos niños o niñas-líderes.

Para darle continuidad a la investigación es necesario, por un lado, retornar a los talleres de proyectos de fin de carrera la información que aquí se obtiene para dar forma a las propuestas de los niños, complementándolas con lo que proponen los otros grupos sociales de cada barrio. De igual forma, hay que continuar realizando los talleres, para poder completar el mapeo y para llegar a conclusiones más contundentes y, sobre todo, definir a la brevedad posible los indicadores y su unidad de medición, que permitan sistematizar la información obtenida.

Por otro lado, debido a las alertas de género declaradas en el estado de Veracruz, consideramos pertinente continuar la investigación en una de las colonias consideradas con más alto grado de percepción de inseguridad, para lo cual nos hemos vinculado con el proyecto Ciudades Seguras y Amables con las Mujeres ${ }^{13}$ donde nuestro aporte será este taller, poniendo especial cuidado en los aspectos de percepción de seguridad/inseguridad por parte de niñas y niños, y sobre todo, en las propuestas que hagan para sentirse más seguros y seguras.

Nos vincularemos también con los talleres Ciudad y Convivencia ${ }^{14}$, que incluyen a todos los actores sociales, ya que consideramos imprescindible complementar la mirada infantil, con la convivencia entre los niños y niñas y todos los otros grupos etarios: jóvenes, adultos y adultos mayores, y con personas de otros grupos sociales.

Finalmente, nos hemos vinculado con la

13 Uno de los 8 proyectos ganadores del Premio a la investigación interdisciplinaria en torno a la Agenda 2030 para el Desarrollo Sostenible de la ONU, Coordinado por Laura Mendoza Kaplan y Harmida Rubio Gutiérrez, UV 2018.

14 Proyecto de vinculación entre la Universidad Veracruzana y la Universidad de Chiba, Japón, desde 2001.17 talleres a la fecha, coordinados por Toshio Kitahara, Donyun Kwak y Laura Mendoza Kaplan. 
Red de Espacios Comunitarios como Nodos de Articulación y Reproducción de la Economía Solidaria en la región Xalapa, que se está construyendo con la Dirección de Desarrollo Económico del H. Ayuntamiento de Xalapa, con la intención concreta de crear o reactivar, según sea el caso, las casas comunitarias o centros de barrio, y creemos necesario incluir ahí los resultados de esta investigación.

\section{Referencias}

Mendez, S. (2018). La diagnosi arquitectònica de l'espai públic a través de la percepció dels infants (Tesis doctoral). Universidad Politécnica de Catalunya, España.

Mendoza, L. (2013). Barrio, Identidad y Convivencia: un proceso formativo transdisciplinario para la proyección de espacios comunitarios (Tesis doctoral). Universidad Nacional Autónoma de México, México.

Mendoza, L. \& Carrillo, K. (Noviembre de 2017). Identidades infantiles: percepción del barrio y la ciudad en niños de diferentes contextos. International Conferences Arquitectonics: Mind, Land, Society. https://pa.upc.edu/ca/ Varis/altres/arqs/congresos /international-conference-arquitectonics-mind-land-society/final-papers/laura-mendoza-kaplan-karla-elisa-carrillo-rodriguez.pdf/ view

Muntañola. J. (1974). La arquitectura como lugar. http:// home.fa.utl.pt/ al7531/ pedidos/livros/Muntanola-Thornberg-La-Arquitectura-Como-Lugar.pdf

Tonucci, F. (2015). La ciudad de los niños. Barcelona: Editorial Graó. 